\title{
Quantitative bi-component T2* analysis of histologically normal Achilles tendons
}

\author{
Eric Y. Chang ${ }^{1,2}$ \\ Jiang $\mathrm{Du}^{2}$ \\ Sheronda Statum ${ }^{2}$ \\ Chantal Pauli ${ }^{3}$ \\ Christine B. Chung ${ }^{1,2}$ \\ 1 Radiology Service, VA San Diego Healthcare Sy- \\ stem, USA \\ 2 Department of Radiology, University of California, \\ San Diego Medical Center, Usa \\ 3 Institute of Surgical Pathology, University Hospital \\ Zurich, Switzerland
}

Corresponding author:

Eric Y. Chang

Radiology Service, VA San Diego Healthcare System

3350 La Jolla Village Drive

92161 San Diego, USA

E-mail: ericchangmd@gmail.com

\section{Summary}

Introduction: the aim of this pilot study was to implement ultrashort echo time (UTE) MRI with bicomponent analysis on grossly normal Achilles tendons with histologic correlation.

Materials and methods: six tendon samples which were grossly normal on visual inspection and palpation were harvested. A 2D UTE pulse sequence was implemented on a $3 T$ MR scanner and bicomponent and single-component $\mathrm{T}^{*}$ analysis was performed. Tendon samples were histologically processed and evaluated.

Results: mean short T2* fraction was $79.2 \%$ (95\% confidence interval $[\mathrm{Cl}], 70.1-88.3 \%$ ), mean short T2* was $1.8 \mathrm{~ms}(95 \% \mathrm{Cl}, 1.3-2.3 \mathrm{~ms})$, mean long T2* fraction was $20.8 \%(95 \% \mathrm{Cl}, 11.7-29.9 \%)$, mean long T2* was 9.2 ms $(95 \%$ Cl, $5.1-13.3 \mathrm{~ms})$, and mean single-component T2* was $2.5 \mathrm{~ms}(95 \%$ $\mathrm{Cl}, 1.8$ - $3.1 \mathrm{~ms})$.

Discussion: 2D UTE MRI with bi-component and single-component $\mathrm{T2}^{*}$ analysis was successfully implemented. Inter-individual variation can be demonstrated in grossly and histologically normal Achilles tendons.

KEY WORDS: tendon, ultrashort TE, bi-component analysis.

\section{Introduction}

The Achilles tendon is composed of approximately $66 \%$ water and the dry weight is approximately $87 \%$ collagen and $0.2 \%$ glycosaminoglycans (GAGs) ${ }^{1,2}$. Historically, knowledge of tendon composition has required invasive techniques such as biopsy or has only been available using post-mortem tissue. However, in the past few years, this information has become available using non-invasive magnetic resonance (MR) imaging techniques.

MR imaging detects the protons in water and can provide quantitative measures in the form of T2 or T2* values, representing intrinsic tissue biomarkers. These values are typically calculated using single-component models, but can also be calculated using a bicomponent model ${ }^{3}$. Bi-component fitting allows for protons to be assigned to "short" and "long" pools, representing water bound to macromolecules (including collagen and glycosaminoglycans) and bulk water, respectively ${ }^{3-5}$.

A new group of clinically compatible sequences, collectively known as ultra short time-to-echo (UTE) techniques ${ }^{6}$, have been used to show statistically significant differences in bi-component T2* values between healthy volunteers and patients with Achilles pathology ${ }^{7}$. However, there is considerable overlap in reported values when studied in vivo. Additionally, in vivo studies have shown regional variation, with varying quantitative values based on sample location in the proximal-distal direction ${ }^{7,8}$ or within a transverse plane ${ }^{8}$, even in the same patient. The reason for these differences is unclear and differences in normal matrix composition may be a contributing factor. However, subclinical Achilles tendon degeneration is common and an alternate possibility. In cadavers that were reported to be healthy at the time of accidental death, Kannus et al. found Achilles tendon degeneration in $35 \%$ of specimens using histologic analysis $^{9}$.

There has been a paucity of studies that have performed correlation between quantitative MR measures and histology using Achilles tendons ${ }^{10,11}$. To the best of our knowledge this has not yet been performed using bi-component analysis with UTE MRI techniques. Therefore, the aim of this pilot study was to implement UTEMRI with bi-component analysis on grossly normal Achilles tendons with histologic correlation to observe the degree of variability. 


\section{Materials and methods}

\section{Sample Preparation}

This research study has been conducted according to the international standard and as required by the journal ${ }^{12}$. Specimens used in this study underwent a single freeze-thaw cycle, which occurred prior to dissection. Six tendon samples were dissected from three donor ankles ( 3 females; $86.3 \pm 0.6$ years, mean \pm standard deviation) and were grossly normal on visual inspection and palpation without evidence for tearing, tendon enlargement, or softening. Tendon samples were harvested from the mid-section, defined as the portion of tendon within 3-4 cm above the calcaneal tuberosity. Each tendon sample was approximately $1.5 \mathrm{~cm}$ in length. Tendon samples were placed into syringes filled with Fomblin (Ausimont, Thorofare, $\mathrm{NJ}$ ) to minimize dehydration and susceptibility effects ${ }^{13}$ and stored in the refrigerator at $4^{\circ} \mathrm{C}$. On the day of imaging, specimens were equilibrated with room temperature for at least 4 hours prior to scanning.

\section{MR imaging and image analysis}

MR imaging was performed on a clinical 3T MR scanner (SignaHDx, GE Healthcare Technologies, Milwaukee, WI). The system had gradients capable of a slew rate of $150 \mathrm{~T} / \mathrm{m} / \mathrm{s}$ and amplitude of 40 $\mathrm{mT} / \mathrm{m}$ on each axis. A 1 -inch diameter transmit-receive birdcage coil was used for signal excitation and reception. Tendons samples were carefully oriented in the same expected position as a clinical scan with the long axis of the Achilles tendon parallel to the main magnetic field. The 2D UTE sequence was performed which uses a short half pulse excitation followed by $2 \mathrm{D}$ radial ramp sampling (minimal nominal TE of $8 \mu \mathrm{s}$ ). Imaging parameters included: TR = $100 \mathrm{~ms}, \mathrm{TE}=0.008,0.1,0.2,0.4,0.6,0.8,2,4,10$, 15,20 , and $30 \mathrm{~ms}$, field-of-view $(\mathrm{FOV})=5 \mathrm{~cm}$, slice thickness $=3 \mathrm{~mm}$, matrix $=256 \times 256$, and number of excitation $(\mathrm{NEX})=2$. Total imaging time was about 13 minutes.

Using the axial images, regions of interest (ROI) were placed within each tendon and copied to the corresponding position on subsequent TE images (Fig. 1). The mean intensity within each ROI was used for curve fitting. Single and bi-component $\mathrm{T}^{*}$ analysis was performed using a semi-automated MATLAB (The Mathworks Inc., Natick, MA) code developed in-house as previously described 3,14 . For singlecomponent analysis, UTE signals $S_{N}(t)$ were fitted with the following equation: $S_{N}(t)=A \times \exp \left(-t / T 2^{*}\right)+$ noise. For bi-component analysis, UTE signals $S_{N}(t)$ were fitted with the following equation: $S_{N}(t)=A_{S} x$ $\exp \left(-\mathrm{TE} / \mathrm{T} 2^{*} \mathrm{~S}\right)+\mathrm{A}_{\mathrm{L}} \mathrm{x} \exp \left(-\mathrm{TE} / \mathrm{T} 2^{*} \mathrm{~L}\right)+$ noise, where $A_{S}$ is the amplitude of the short component, $A_{L}$ is the amplitude of the long component, $\mathrm{T}^{*}{ }^{*} \mathrm{~S}$ is the short component $\mathrm{T}^{*}$, and $\mathrm{T}^{*}{ }^{*} \mathrm{~L}$ is the long component $\mathrm{T}_{2}{ }^{*}$. Apparent short component fraction was defined as
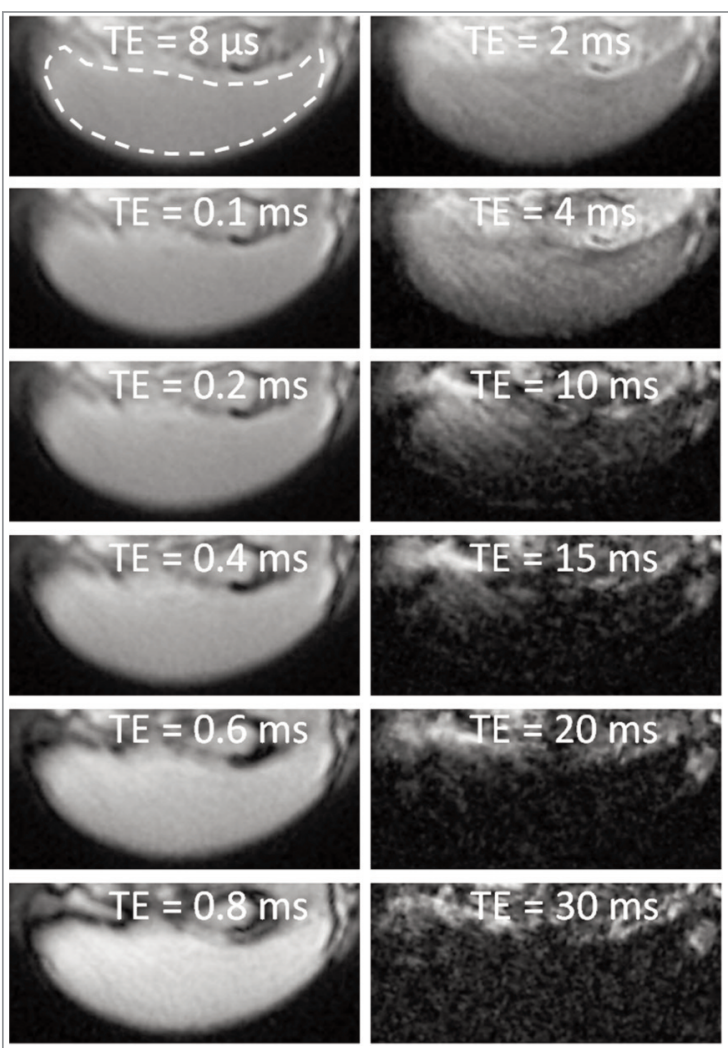

Figure 1. 2D UTE MR images of Achilles tendon, sample 5, shows signal decay with increasing echo time (TE). Region of interest used for quantitative analysis is also shown (dashed white line).

$A_{S} /\left(A_{S}+A_{L}\right)$. Estimation of background noise was performed using a maximum likelihood estimation distribution fitting of a partial histogram and non-negative least square curve fitting was employed for both single and bi-component models. Fit curves along with 95\% confidence intervals and residual signal curves were created ${ }^{15}$. Coefficient of determination $\left(R^{2}\right)$ and root mean squared error (RMSE) were calculated to quantify the goodness of $\mathrm{fit}^{8,16}$. Single component $\mathrm{T}^{*}$ maps were calculated by a pixel-by-pixel basis for visual comparison to histologic slides.

\section{Histologic analysis}

Each Achilles tendon piece was fixed in 4\% formalin, dehydrated with alcohol, and embedded in paraffin. Sections were cut and stained with hematoxylin and eosin (H\&E) and Safranin-O-Fast Green (Saf-O) to evaluate for collagen structural integrity and proteoglycan content, respectively ${ }^{17}$. Tendons were evaluated for signs of tendinosis, including abnormal tenocyte morphology, chondroid metaplasia, fatty/mucoid degeneration, or proteoglycan/GAG deposition ${ }^{9,18}$. All sections were examined by an experienced pathologist with expertise in orthopedic pathology. 


\section{Statistical analysis}

Descriptive statistics were performed. Mean values with standard deviation (SD) and 95\% confidence intervals $(\mathrm{Cl})$ were reported for single and bi-component analyses. Statistical analyses were performed using the SPSS software package (version 21; SPSS, Chicago, IL, USA).

\section{Results}

The 2D UTE protocol generated high quality MR images that adequately sampled the signal decay pattern of tendon (Fig. 1). Bi-component curve fitting was superior compared with the single-component algorithm for all tendons (Fig. 2), and the goodness of fit quantifiers ( $R^{2}$ and RMSE) were also lower for the bi-component fit compared with single-component fitting. Quantitative results are listed in Table 1. Mean short

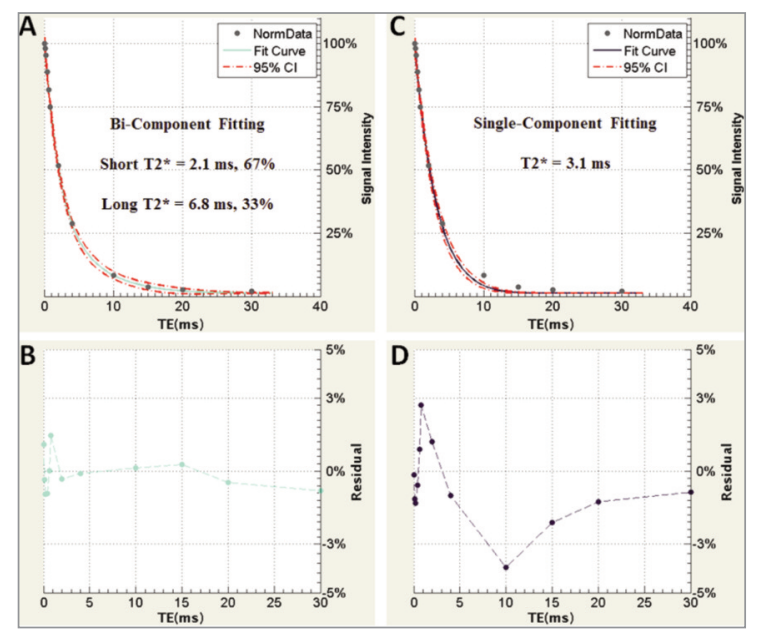

Figure 2. Quantitative analysis of Achilles tendon, sample 5. Signal fitting with a bi-component algorithm (A) shows superior fitting compared with the single-component algorithm $(C)$. This is confirmed with systemic residual signal plot which is smaller with the bi-component algorithm (B) compared with the single algorithm (D). Additionally, coefficient of determination $\left(R^{2}\right)$ /root mean squared error (RM$\mathrm{SE}$ ) were was 1.0/23.0 for the bi-component fit compared with $1.0 / 49.7$ for the single-component fit.
T2* fraction was $79 \%$ (range $67-93 \%$ ), mean short $\mathrm{T}^{*}$ was $1.8 \mathrm{~ms}$ (range 1.4-2.4 ms), mean long T2* fraction was $21 \%$ (range $8-33 \%$ ), mean long $\mathrm{T}^{*}$ was $9.2 \mathrm{~ms}$ (range 5.6-16.4 ms), and mean single-component T2* was $2.5 \mathrm{~ms}$ (range 1.9-3.1 ms).

Single-component $\mathrm{T}^{*}$ maps showed regions of variation within a transverse slice, however all tendons were normal on histologic evaluation without gross discernable differences on either stain (Figs. 3, 4). Specifically, there were no regions of collagen fiber
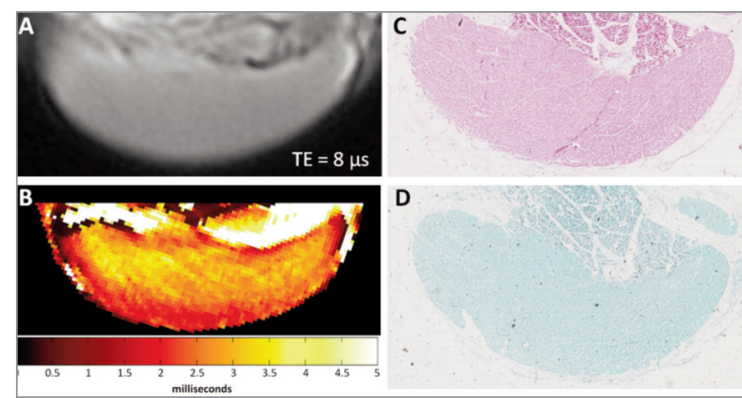

Figure 3. UTE MRI and histologic analysis of Achilles tendon, sample 5. 2D UTE MR image with $8 \mu \mathrm{s}$ TE (A), singlecomponent $\mathrm{T}^{*}$ map (B), H\&E stain (C), and Saf-O stain (D) shows normal tendon. Regional variability is seen on the $\mathrm{T}^{*}$ map (B), characterized by increased $\mathrm{T} 2^{*}$ centrally, which is not evident on histological stains.

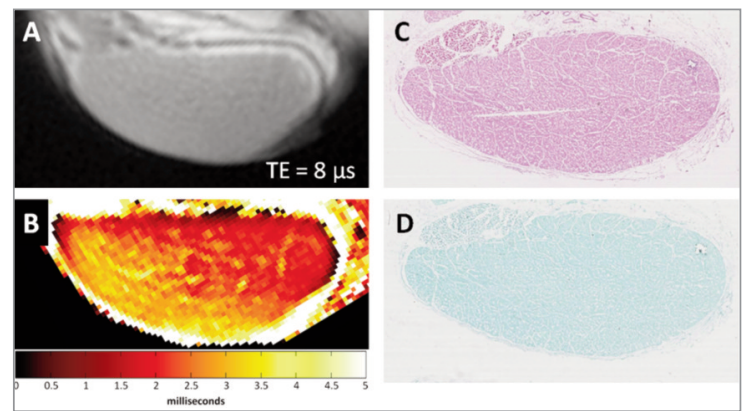

Figure 4. UTE MRI and histologic analysis of Achilles tendon, sample 6. 2D UTE MR image with $8 \mu \mathrm{s}$ TE (A), singlecomponent $\mathrm{T}^{*}$ map (B), H\&E stain (C), and Saf-O stain (D) shows normal tendon. Regional variability is seen on the $\mathrm{T}^{*}$ map $(\mathrm{B})$, characterized by increased $\mathrm{T} 2^{*}$ on the left side of the image,which is not evident on histological stains.

Table 1. Bi-component and single component T2* analysis results for normal Achilles tendons.

\begin{tabular}{llllllll}
\hline $\begin{array}{l}\text { Sample } \\
\text { Number }\end{array}$ & $\begin{array}{l}\text { Short T2 } \\
\text { Fraction }(\%)\end{array}$ & $\begin{array}{l}\text { Short T2 } \\
(\mathrm{ms})\end{array}$ & $\begin{array}{l}\text { Long T2 } \\
\text { Fraction }(\%)\end{array}$ & $\begin{array}{l}\text { Long T2* } \\
(\mathrm{ms})\end{array}$ & $\begin{array}{l}\mathrm{R}^{2} / \mathrm{RMSE} \\
(\mathrm{Bi}-\text {-component) }\end{array}$ & $\begin{array}{l}\text { Single-component } \\
\text { T2* }\end{array}$ & $\begin{array}{l}\mathrm{R}^{2} / \mathrm{RMSE} \\
\text { (Single-component) }\end{array}$ \\
\hline 1 & 73.8 & 1.4 & 26.2 & 5.6 & $1.0 / 36.7$ & 2.0 & $1.0 / 61.6$ \\
2 & 82.9 & 1.4 & 17.1 & 10.2 & $1.0 / 21.8$ & 1.9 & $1.0 / 53.5$ \\
3 & 82.2 & 1.4 & 17.8 & 7.0 & $1.0 / 34.0$ & 1.9 & $1.0 / 55.7$ \\
4 & 76.2 & 2.2 & 23.8 & 9.2 & $1.0 / 41.3$ & 3.1 & $1.0 / 69.4$ \\
5 & 67.4 & 2.1 & 32.6 & 6.8 & $1.0 / 23.0$ & 3.1 & $1.0 / 49.7$ \\
6 & 92.5 & 2.4 & 7.5 & 16.4 & $1.0 / 18.6$ & 2.7 & $1.0 / 32.5$ \\
Mean \pm SD & $79.2 \pm 8.7$ & $1.8 \pm 0.5$ & $20.8 \pm 8.7$ & $9.2 \pm 3.9$ & & $2.5 \pm 0.6$ & \\
{$[95 \% \mathrm{Cl}]$} & {$[70.1-88.3]$} & {$[1.3-2.3]$} & {$[11.7-29.9]$} & {$[5.1-13.3]$} & {$[1.8-3.1]$} & \\
\hline
\end{tabular}


disruption, abnormal tenocyte morphology, metaplasia or degeneration. No regions of abnormal staining were noted on Saf-O to suggest degenerative proteoglycan/GAG deposition.

\section{Discussion}

In our pilot study we have shown that UTE MRI with bi-component and single-component $\mathrm{T}^{*}$ analysis can be successfully implemented, and bi-component analysis shows superior curve fitting relative to single-component analysis. Additionally, we found that a range of quantitative values can be seen in grossly and histologically normal Achilles tendons. De Mos et al. have previously shown that normal Achilles tendons can vary in water content with a $95 \%$ confidence interval of $55.4-77.0 \%$ (mean $66.2 \%$ ) as well as dry $\%$ collagen content with a 95 confidence interval of $55.5-73.5 \%$ (mean $64.5 \%$ ), using weights and high-performance liquid chromatography, respectively ${ }^{1}$. Using clinically compatible UTE techniques, we show that similar variation is detectable using MRI on histologically similar samples evaluated with H\&E and SafO stains.

Other Authors have also reported a range of bi-component and single-component $\mathrm{T}^{*}$ analysis values, and our results are within range to previously published studies. For instance, Juras et al. studied 10 healthy volunteers using 3D-UTE with bi-component analysis and for their results at 3T, mean short T2* fractions varied from $47-79 \%$, depending on sampling location within the Achilles tendon ${ }^{8}$. Short T2* fractions in our sample demonstrated overlap with this range, varying from $67-93 \%$. Similarly, in the same study, Juras et al. ${ }^{8}$ reported mean long T2* values to range from 7.9-31.8 $\mathrm{ms}$ which compares to our long $\mathrm{T}^{*}$ values which range from 5.6-16.4 ms. Regarding single-component analyses, the range of our $\mathrm{T}^{*}$ values $(1.9-3.1 \mathrm{~ms})$ is similar to that previously reported by Filho et al. (1.8-2.6 ms $)^{10}$.

From a clinical perspective, the wide range of values in our sample and in the literature confirms the important point that caution must be made when interpreting quantitative MRI values and utilizing a cut-off value for the determination of normal versus abnormal tissue ${ }^{19,20}$. In particular, overlap of quantitative values between normal and abnormal tissues can degrade diagnostic performance, even in spite of statistical significance between groups ${ }^{19,20}$. However, non-invasive quantitative MRI techniques may be particularly useful for longitudinal measurements in individuals, such as for monitoring after treatment or therapy.

Using the conventional light microscopy techniques in our study, we were unable to determine the source of individual variability since the tendons appeared normal and histologically similar on H\&E and Saf-O stains. However, the inter-specimen variability can be not only due to water and collagen content, but differences in collagen fiber orientation. Under polarized light microscopy, tendon collagen fibers have been shown to run in longitudinal, transverse and oblique directions and can form complex patterns, including spirals and plaits ${ }^{21}$. It is well known that tendon is exquisitely sensitive to relatively small changes in orientation between its long axis and the direction of the main magnetic field 22,23 . Wang et al. have shown that 10-15 degree differences in orientation can alter not only measurements in relaxation times of individual components, but also the number of measureable components $^{24}$. As discussed by Zheng et al., transverse relaxation measurements are actually "composite" measures reflecting complex factors at both molecular/structural levels within a specimen and procedural levels including instrumentation used for measurements/data analysis ${ }^{25}$. The complex interplay between detectable relaxation differences, the physical reason for these differences, and the clinical significance of these differences deserves additional study.

Our study has some limitations. First, our sample size was small, consisting of six tendons from three donors. However, this was a pilot study, implementing a relatively new quantitative MRI technique. We were able to confirm that individual variability could be demonstrated in grossly and histologically similar Achilles tendons. With more specimens we would expect an even greater amount of variability. Second, we used only routine light microscopic methods with $\mathrm{H} \& \mathrm{E}$ and Saf-O staining for histologic analysis. Other techniques including polarized light microscopy, transmission and scanning electronic microscopy, and histochemical techniques may have demonstrated additional variability in tissue. However, the basic diagnosis of tendon degeneration can be determined with routine light microscopy ${ }^{9}$.

In conclusion, in our pilot study, we have found that UTE MRI with bi-component and single-component $\mathrm{T}^{*}$ analysis can be successfully implemented. Additionally, we found that grossly and histologically normal Achilles tendons can demonstrate a range of quantitative values using single and bi-component $\mathrm{T}^{*}$ analysis, reflecting individual variability.

\section{Acknowledgements}

The Authors gratefully acknowledge grant support from the VA Clinical Science Research and Development Service (Career Development Award IK2CX000749).

\section{References}

1. de Mos M, van E1 B, DeGroot J, et al. Achilles tendinosis: changes in biochemical composition and collagen turnover rate. Am J Sports Med. 2007;35(9):1549-1556.

2. Amiel D, Frank C, Harwood F, Fronek J, Akeson W. Tendons and ligaments: a morphological and biochemical comparison. J Orthop Res. 1984;1(3):257-265.

3. Du J, Diaz E, Carl M, Bae W, Chung CB, Bydder GM. Ultrashort echo time imaging with bicomponent analysis. Magn $\mathrm{Re}$ son Med. 2012;67(3):645-649. 
4. Henkelman RM, Stanisz GJ, Kim JK, Bronskill MJ. Anisotropy of NMR properties of tissues. Magn Reson Med. 1994; 32(5):592-601.

5. Peto S, Gillis P, Henri VP. Structure and dynamics of water in tendon from NMR relaxation measurements. Biophys $\mathrm{J}$. 1990;57(1):71-84.

6. Chang EY, Du J, Chung CB. UTE imaging in the musculoskeletal system. J Magn Reson Imaging. 2014.

7. Juras $\mathrm{V}$, Apprich S, Szomolanyi P, Bieri O, Deligianni X, Trattnig S. Bi-exponential T2 analysis of healthy and diseased Achilles tendons: an in vivo preliminary magnetic resonance study and correlation with clinical score. Eur Radiol. 2013;23(10):2814-2822.

8. Juras V, Zbyn S, Pressl C, et al. Regional variations of $\mathrm{T}(2)^{*}$ in healthy and pathologic achilles tendon in vivo at 7 Tesla: preliminary results. Magn Reson Med. 2012;68(5):1607-1613.

9. Kannus $P$, Jozsa L. Histopathological changes preceding spontaneous rupture of a tendon. A controlled study of $891 \mathrm{pa}-$ tients. J Bone Joint Surg Am. 1991;73(10):1507-1525.

10. Filho GH, Du J, Pak BC, et al. Quantitative characterization of the Achilles tendon in cadaveric specimens: $\mathrm{T} 1$ and $\mathrm{T}^{*}$ measurements using ultrashort-TE MRI at 3 T. AJR Am J Roentgenol. 2009;192(3):W117-124.

11. Juras V, Apprich S, PressI C, et al. Histological correlation of 7 T multi-parametric MRI performed in ex-vivo Achilles tendon. Eur J Radiol. 2013;82(5):740-744.

12. Padulo J, Oliva F, Frizziero A, Maffulli N. Muscles, Ligaments and Tendons Journal. Basic principles and recommendations in clinical and field science research. MLTJ. 2013;4:250-252.

13. Chang EY, Du J, Bae WC, Statum S, Chung CB. Effects of achilles tendon immersion in saline and perfluorochemicals on T2 and T2*. J Magn Reson Imaging. 2014;40(2):496-500.

14. Diaz E, Chung CB, Bae WC, et al. Ultrashort echo time spectroscopic imaging (UTESI): an efficient method for quantifying bound and free water. NMR Biomed. 2012;25(1):161-168.
15. Biswas R, Bae W, Diaz E, et al. Ultrashort echo time (UTE) imaging with bi-component analysis: bound and free water evaluation of bovine cortical bone subject to sequential drying. Bone. 2012;50(3):749-755.

16. Juras V, Zbyn S, Szomolanyi P, Trattnig S. Regression error estimation significantly improves the region-of-interest statistics of noisy MR images. Med Phys. 2010;37(6):2813-2821.

17. Pauli C, Grogan SP, Patil S, et al. Macroscopic and histopathologic analysis of human knee menisci in aging and osteoarthritis. Osteoarthritis Cartilage. 2011;19(9):1132-1141.

18. Jarvinen $M$, Jozsa L, Kannus $P$, Jarvinen $T L$, Kvist M, Leadbetter W. Histopathological findings in chronic tendon disorders. Scand J Med Sci Sports. 1997;7(2):86-95.

19. Spencer RG, Lukas VA, Cortese BD, et al. Clinical Decision Rules for Detection of Cartilage Degradation Based on Univariate MR Parameter Analysis. Proc Intl Soc Mag Reson Med. 2014. ISMRM 22th Scientific Meeting \& Exhibition. Milan, Italy; Abstract 301.

20. Spencer RG, Pleshko N. How Do Statistical Differences in Matrix-sensitive Magnetic Resonance Outcomes Translate Into Clinical Assignment Rules? J Am Acad Orthop Surg. 2013; 21(7):438-439.

21. Kannus $P$. Structure of the tendon connective tissue. Scand J Med Sci Sports. 2000;10(6):312-320.

22. Fullerton GD, Rahal A. Collagen structure: the molecular source of the tendon magic angle effect. J Magn Reson Imaging. 2007;25(2):345-361.

23. Krasnosselskaia LV, Fullerton GD, Dodd SJ, Cameron IL. Water in tendon: orientational analysis of the free induction decay. Magn Reson Med. 2005;54(2):280-288

24. Wang N, Xia Y. Anisotropic analysis of multi-component T2 and T1 rho relaxations in achilles tendon by NMR spectroscopy and microscopic MRI. J Magn Reson Imaging. 2013;38(3):625633.

25. Zheng S, Xia Y. Multi-components of T2 relaxation in ex vivo cartilage and tendon. J Magn Reson. 2009;198(2):188-196. 